\title{
Educação e Trabalho Interprofissional na Atenção ao Transtorno do Espectro do Autismo: Uma Necessidade para a Integralidade do Cuidado no SUS
}

\author{
Samantha Maranhão, ${ }^{1}$ Lilian Lisboa, ${ }^{2}$ Celina Reis, ${ }^{3}$ Reginaldo Freitas Júnior ${ }^{4}$
}

\begin{abstract}
RESUMO
Objetivo: Trata-se de um relato de experiência na área da saúde e educação, cujo objetivo é documentar e refletir a relação entre educação e trabalho interprofissional na atenção ao Transtorno do Espectro do Autismo (TEA). Método: Localizado no município de Macaíba, no Rio Grande do Norte, o Serviço de Atenção ao TEA (Satea) está inserido no Sistema Único de Saúde (SUS) como referência ambulatorial para a atenção à saúde materno-infantil e para reabilitação auditiva, motora e intelectual. A instituição do Satea veio com a finalidade de atuar na formação, desenvolvimento e educação permanente de profissionais de saúde, desenvolvendo ações integradas de ensino, pesquisa e extensão centradas nas concepções de responsabilidade social, equidade, qualidade e eficiência, para gerar evidências científicas, desenvolver estratégias e promover parcerias capazes de fortalecer e auxiliar o SUS. Resultado: O Satea vem se constituindo como serviço de referência local ao atendimento de crianças com TEA. Muito deste reconhecimento advém de um trabalho pautado pela interprofissionalidade e pela construção de práticas colaborativas com as equipes locais de Estratégia de Saúde da Família. Esta experiência tem favorecido o acesso dos profissionais locais à educação permanente, reconhecendo-os na condição de agentes fundamentais para o aprendizado em serviço e para a legitimação do papel formador do SUS. Conclusão: Compreende-se que as experiências apresentadas neste trabalho podem servir de base para outros serviços especializados na saúde materno-infantil e na reabilitação, além de oferecer aos usuários do SUS a documentação do processo de criação e adoção de um serviço especializado no atendimento do TEA.
\end{abstract}

Palavras-chave: Transtornos do neurodesenvolvimento. Transtorno autístico. Integralidade em saúde. Educação em saúde.

INTERPROFESSIONAL EDUCATION AND WORK IN ATTENTION TO AUTISM SPECTRUM DISORDER

\section{ABSTRACT}

Objective: This is an account of experience in health and education, whose objective is to document and reflect the relationship between education and interprofessional work in the attention to Autism Spectrum Disorder (ASD). Method: Located in the city of Macaíba, in Rio Grande do Norte, the TEA Attention Service (Satea) is inserted in the Unified Health System (SUS) as an outpatient reference for maternal and child health care and for auditory, motor and rehabilitation and intellectual. The implementation of Satea came with the prerogative to act in the training, development and permanent education of health professionals, developing integrated teaching, research and extension actions focused on conceptions of social responsibility, equity, quality and efficiency, to generate scientific evidence, develop strategies and promote partnerships capable of strengthening and assisting SUS. Outcome: Satea is becoming a local reference service for the care of children with ASD. Much of this recognition comes from a work based on interprofessionality and the construction of collaborative practices with the local Teams of Family Health Strategy. This experience has favored the access of the local professionals to the permanent education, recognizing them as fundamental agents for the learning in service and for the legitimation of the formative role of SUS. Conclusion: It is understood that the experiences presented in this study can serve as a basis for other specialized services in maternal and child health and rehabilitation; in addition to offering SUS users the documentation of the implementation process and implementation of a specialized service in TEA.

Keyword: Neurodevelopmental disorders. Autistic disorder. Integrality in health. Health education.

\footnotetext{
${ }^{1}$ Neuropsicóloga, preceptora multiprofissional do Centro de Educação e Pesquisa em Saúde Anita Garibaldi, Instituto Santos Dumont. samantha.maranhao@isd.org.br ${ }^{2}$ Fisioterapeuta. Gerente do Centro de Educação e Pesquisa em Saúde Anita Garibaldi, Instituto Santos Dumont. lilian.lisboa@isd.org.br

${ }^{3}$ Médica neurologista infantil, preceptora médica do Centro de Educação e Pesquisa em Saúde Anita Garibaldi, Instituto Santos Dumont. celina.reis@isd.org.br ${ }^{4}$ Diretor-Geral do Instituto Santos Dumont. reginaldo@isd.org.br
} 


\section{INTRODUÇÃO}

O Transtorno do Espectro do Autismo (TEA) constitui-se como Transtorno do Neurodesenvolvimento que atinge quase 2 milhões de brasileiros. A Organização das Nações Unidas (ONU) estima que existam 70 milhões de pessoas com autismo, cerca de $1 \%$ da população mundial. Em estudo epidemiológico realizado no ano de 2012, 98.367 pessoas foram identificadas com TEA na Região Nordeste do país, e destas, apenas 393 têm acesso à serviços de saúde (MELLO, 2013).

O TEA caracteriza-se pela presença de uma díade de comprometimentos qualitativos nos domínios da interação/comunicação social e padrões comportamentais (AMERICAN..., 2014). Sinais de alerta já são passíveis de identificação nos seis primeiros meses de vida da criança. Conforme Gillet (2014), os sinais de autismo podem mudar consideravelmente com a idade, apresentando-se mais sutis nos 6 primeiros meses e mais aparentes a partir dos 18-24 meses. Embora não haja, até o momento, evidência que respalde um diagnóstico clínico no primeiro ano de vida da criança, é crescente o volume de conhecimento sugerindo a possibilidade de identificação de sinais de risco do autismo nesse período (AMERICAN..., 2014).

A díade de comprometimentos qualitativos nos domínios da interação/comunicação social e padrões comportamentais no TEA tem curso evolutivo crônico, portanto o diagnóstico e tratamento precoces são fundamentais para a proposição de intervenções que garantam o aprimoramento do neurodesenvolvimento infantil e, por conseguinte, uma melhor qualidade de vida para a criança autista e seus familiares.

Embora existam critérios clínicos que definam o diagnóstico de TEA, a etiologia multifatorial e, até o momento, a ausência de marcadores biológicos diagnósticos potencializam a necessidade de desenvolvimento de iniciativas de trabalho interprofissional e práticas colaborativas com as Estratégias de Saúde da Família a fim de facilitar a identificação de sinais e sintomas de risco clínico de TEA, para, consequentemente, promover a intervenção precoce (antes dos 5 anos de idade).

O diagnóstico e intervenção precoces (antes dos 5 anos) são fundamentais para o neurodesenvolvimento infantil e, por conseguinte, para uma melhor condição de vida da criança e seus familiares. Os estudos mais recentes sobre autismo apontam que intervenções precoces são as mais efetivas e podem di- minuir a necessidade de assistência especial para as faixas de idade seguintes (DAVID; BABINEAU; WALL, 2016; GARCIA; LAMPREIA, 2011).

Chinello, Gangi e Valenza (2018) estudaram o desenvolvimento motor típico de lactentes de 12 a 17 meses e observaram o progresso gradativo de substituição dos reflexos primitivos pela aquisição de ações motoras direcionadas, especialmente ações com a mão ou boca. $O$ estudo sugere que os lactentes com indicadores clínicos de risco para o desenvolvimento infantil, sem intervenção precoce, frequentemente possuem atraso na aquisição de movimentos especializados, devido à persistência de reflexos primitivos.

De modo similar, Srinivasan e Bhat (2016) compararam a habilidade de compartilhar objetos, ao engatinhar e andar, em grupo de 16 crianças entre 9 e 15 meses com desenvolvimento típico e com risco para o desenvolvimento infantil. De forma geral, os bebês com risco obtiveram pior desempenho na habilidade de compartilhar objetos com cuidadores. Eles frequentemente demonstraram menores taxas de passos (durante o andar) em direção aos alvos apropriados para a tarefa, ou seja, cuidadores ou objetos.

Desde o nascimento, o cérebro humano possui zonas corticais funcionais, especialmente dedicadas à apreciação visual do rosto humano (circunvolução fusiforme do hemisfério direito) e à apreciação auditiva da palavra. Falhas na percepção facial e vocal repercutem na resposta-sorriso do bebê, que, por sua vez, podem repercutir no reconhecimento, entendimento e compartilhamento de suas emoções primárias (alegria, tristeza, medo, raiva, nojo). Falhas na percepção facial e vocal também parecem repercutir na imitação bucofacial do outro (ZILBOVICIUS et al., 2006).

Nessa direção, Pierce et al. (2011) observaram o direcionamento do olhar de crianças entre 17 e 24 meses com TEA e verificaram preferência de direcionamento visual para imagens de formas geométricas em movimento, bem como atípica exploração visual de rostos. Nogueira, Seabra e Moura (2000 como citado em BOSA, 2002, p. 84) apresentam achados de bebês com suspeita de autismo que possuem menor frequência de contato visual e nenhum episódio de interação com sua mãe. Recém-nascidos, com apenas horas de vida, conseguem imitar o adulto quando este abre a boca e estende a língua para fora. Quando mais velhas, as crianças com desenvolvimento típico direcionam o olhar ou demonstram tendência em alternar entre a pessoa e o objeto de interesse. Aos 12 meses de vida as crianças, tipicamente, olham para o parceiro, após apontar. Aos 14 meses o ato de apontar acompanha o olhar para o parceiro e, aos 16 meses, o 
olhar precede o gesto, sugerindo progressos na habilidade de compreender a importância de tal gesto ao direcionar o olhar do parceiro para o seu próprio foco de interesse (ABREU; CARDOSO-MARTINS; BARBOSA, 2014).

Estudos de vídeos familiares retrospectivos mostram que crianças com risco de TEA dificilmente imitavam as posturas orofaciais e corporais do outro, os movimentos arbitrários das mãos (como abri-las e fechá-las), o apontar direcionado para os objetos, ou ainda, os gestos que imitam a utilização de um objeto (BOSA, 2002). No neurodesenvolvimento de crianças com risco de TEA parece existir uma mudança de caminho no percurso de habilidades que guiam o desenvolvimento social. Schmidt (2017) sugere a diminuição do tempo de fixação do olhar social em bebês entre dois e seis meses de vida com risco de TEA e tal queda parece interferir na robustez de comportamentos sociais imprescindíveis para comunicação e interação social.

Por meio de observações clínicas sistemáticas e longitudinais, Zaqueu et al. (2015) compararam o desenvolvimento social de crianças típicas e com risco de TEA. Eles constataram a presença de falhas gestuais nos primeiros meses de vida a partir da observação do modo pelo qual os bebês com risco olhavam para os objetos, pediam o que desejavam, ou como reagiam quando apontavam para alguma direção. Tais bebês apresentaram sinais clínicos mais evidentes de TEA após os dois anos e meio de idade.

Outro comportamento indicativo de risco para o TEA é a aceitação do colo de qualquer pessoa. Comumente, a partir dos oito meses, a criança tende a estranhar quem não é do seu convívio e até chora, demonstrando aparente insatisfação. Uma criança com risco de TEA sugere sentir-se confortável com qualquer pessoa. $O$ choro quase ininterrupto, uma inquietação constante, ou ao contrário, uma aparente apatia exacerbada, também despertam a atenção. É importante observar, inclusive, se o bebê se incomoda com o toque, com alguns sons e com certas texturas de alimentos (o que comumente dificulta a transição do leite materno para as comidas sólidas). Quando as crianças estão mais velhas os pais geralmente observam ausência da fala, uma aparente surdez e os movimentos pendulares estereotipados de tronco, mãos e cabeça (ZAQUEU et al., 2015).

Desde os primeiros anos de vida as crianças com risco de TEA comumente revelam aparente ausência de necessidade de aconchego, ausência de elevação dos braços (como antecipação a ser pego no colo), falta de imitação da fala ou dos gestos, falha em apontar ou mostrar objetos para os outros e peculiar contato visual. Geralmente essas crianças procuram o objeto/evento de interesse, mas não há partilha social do mesmo. Frequentemente demonstram não responder ao próprio nome, especialmente quando há envolvimento excessivo no que estão fazendo (ABREU; CARDOSO-MARTINS; BARBOSA, 2014).

De modo geral, o conjunto de estudos de neuroimagem sugere que as disfunções neurológicas de crianças com TEA iniciam-se antes mesmo da idade típica do diagnóstico clínico, todavia comumente a metodologia de identificação precoce (antes dos 5 anos) depende de acesso à tecnologia de ponta, inacessível à população em geral. Conforme Schmidt (2017), atualmente há o desafio de converter as soluções de identificação precoce para a saúde pública brasileira.

Além deste desafio, Mello (2013) observa que apenas $15,45 \%$ de crianças entre 0 e 5 anos com diagnóstico ou hipótese de TEA são assistidas no Brasil. Tal realidade brasileira depara-se com a árdua tarefa de identificação clínica ainda na primeira infância e com a heterogeneidade sintomática presente no espectro do autismo. A identificação de sinais e sintomas de risco clínico de TEA requer uma equipe interprofissional especializada, apta a proporcionar procedimentos de diagnóstico-padrão robustos e ações de educação permanente em saúde com a atenção básica.

A Organização Mundial da Saúde (OMS) define interprofissionalidade como ação de educação ou trabalho em que estudantes ou profissionais de dois ou mais cursos, ou núcleos profissionais, aprendem sobre os outros, com os outros e entre si. Equipes de saúde de diferentes núcleos profissionais, que trabalham na perspectiva interprofissional, apresentam maior compreensão das habilidades dos membros que as compõem, logo, compartilham e gerenciam melhor os casos (ARAÚJO et al., 2017).

A complexidade presente na identificação e tratamento do TEA requer um trabalho em equipe pautado em três pilares: 1 ) reflexão sobre os papéis profissionais complementares; 2 ) resolução de problemas em equipe e 3) negociação nos processos decisórios de forma dialógica e com respeito às diferenças dos diversos núcleos de saberes e práticas profissionais. Nessa perspectiva, a interprofissionalidade se constitui como estratégia de trabalho coletivo efetivo, no qual a comunicação e a tomada de decisão em equipe oportuniza a integralidade do cuidado ao TEA (BRASIL, 1988).

A Constituição da República Federativa do Brasil de 1988 determina, em seu artigo 196, que a saúde é um direito de todos e um dever do Estado, garantido 
mediante políticas sociais e econômicas que visem à redução do risco de doença e de outros agravos e ao acesso universal e igualitário às ações e aos serviços para a sua promoção, proteção e recuperação (BRASIL, 1988). No ordenamento jurídico brasileiro existe o Decreto ${ }^{\circ} 8.368$, de 2 de dezembro de 2014, que tem por objetivo garantir à pessoa do espectro autista o direito à saúde no âmbito do Sistema Único de Saúde (SUS), respeitando-se suas especificidades.

$O$ atendimento a tal Decreto exige como etapa primeira a identificação da população clínica, notadamente em fases precoces do desenvolvimento, processo esse que pode ser realizado por uma equipe interprofissional de saúde. De acordo com Surjus (2015, p. 48), "A detecção precoce para o risco do TEA é um dever do Estado, pois, em consonância com os Princípios da Atenção Básica, contempla a prevenção de agravos, promoção e proteção à saúde, propiciando a atenção integral, impactando na qualidade de vida das pessoas e de suas famílias".

Para melhor entendimento, pontua-se que as diretrizes do SUS preconizam a essencialidade de políticas de prevenção e intervenções para crianças em situações de risco e vulnerabilidade, entre as quais, crianças com risco de TEA. O SUS atua de forma estruturada, norteando as ações em todos os níveis de atenção por meio de Redes de Atenção à Saúde, cujos arranjos organizativos de ações e serviços de diferentes densidades tecnológicas devem agir de forma integrada, recebendo apoio técnico e logístico para garantir a integralidade do cuidado (ALEIXO, 2013).

A Estratégia de Saúde da Família (ESF) constitui-se como reorientação do modelo assistencial operacionalizado mediante a criação de equipes multiprofissionais em unidades básicas de saúde. Estas equipes são responsáveis pelo acompanhamento de um número definido de famílias, localizadas em uma área geográfica delimitada. As equipes atuam com ações de promoção da saúde, prevenção, recuperação, reabilitação de doenças e agravos mais frequentes e na manutenção da saúde dessa comunidade (CAMARGO-BORGES; CARDOSO, 2005).

Segundo Camargo-Borges e Cardoso (2005), a ESF constitui-se como porta de entrada para a Rede SUS e visa a reestruturar o modelo de atenção voltado para a integralidade e qualidade da assistência em saúde. Isso posto, capacitar as equipes de Agentes Comunitários de Saúde (ACS) e enfermeiros, em termos de habilidades e competências para a detecção de sinais comportamentais de risco para o autismo, pode influenciar diretamente no encaminhamento adequado para diagnóstico precoce. Conforme Lance- tti (2008), os ACS são potenciais desenvolvedores de um trabalho afetivo. Eles estabelecem um elo entre a comunidade e a equipe de ESF com uma atuação necessariamente relacional. Imbuídos da prerrogativa de pertencer tanto à comunidade quanto à organização sanitária, os agentes podem operar tanto na via da fiscalização dos riscos e controle da vida da população como na criação de redes microssociais de comunicação e troca.

Nesse sentido, o objetivo deste artigo é relatar a experiência de iniciativa de trabalho interprofissional do Serviço de Atenção ao Transtorno do Espectro do Autismo do Centro de Educação e Pesquisa em Saúde Anita Garibaldi, Instituto Santos Dumont, localizado no município de Macaíba (Rio Grande do Norte - Brasil), com ênfase a práticas colaborativas com as Estratégias de Saúde da Família do município. Este relato de experiência objetiva documentar e refletir práticas de integralidade ao cuidado no processo de identificação e intervenção precoce do TEA.

Do ponto de vista metodológico, trata-se de um relato de experiência construído a partir do registro das etapas de criação e instituição do Serviço de Autismo como serviço de referência para o atendimento de pessoas com TEA no Rio Grande do Norte.

\section{PROCESSO DE INSTITUIÇÃO DO SERVIÇO DE ATENÇÃO AO TRANSTORNO DO ESPECTRO DO AUTISMO (SATEA)}

No atual contexto da atenção integral à saúde das pessoas com TEA no Nordeste brasileiro, é inconteste a necessidade de serviços especializados capazes de promover a articulação para integração apropriada do primeiro nível de atenção com os níveis secundários e terciários e com um apropriado balanço de disciplinas profissionais necessários para servir às necessidades de saúde das pessoas.

Quanto ao atendimento ambulatorial de pessoas com TEA, Mello (2013) argumenta que seriam necessárias 40 mil instituições brasileiras especializadas no atendimento de pessoas com Transtornos do Neurodesenvolvimento. Na região Nordeste do Brasil há 13 instituições especializadas para o TEA, quando seriam necessárias 3.254 instituições aptas ao atendimento das 98.367 pessoas identificadas com TEA. Nesse contexto de escassos serviços de referência, o Satea foi instituído no plano tático de 2016 do Centro de Saúde Anita Garibaldi, do qual faz parte como atividade de atendimento interprofissional para crianças e adolescentes do Estado do Rio Grande do Norte com hipótese ou diagnóstico de TEA. 
Localizado no município de Macaíba, no Rio Grande do Norte, o Satea está inserido no SUS como serviço de referência ambulatorial para a atenção à saúde materno-infantil e para habilitação/reabilitação auditiva, motora e intelectual, na proposta de Educação Baseada na Comunidade.

A criação do Satea veio com a prerrogativa de atuar na formação, desenvolvimento e educação permanente de profissionais de saúde, desenvolvendo ações integradas de ensino, pesquisa e extensão centradas nas concepções de responsabilidade social, equidade, qualidade e eficiência, para gerar evidências científicas, desenvolver estratégias e promover parcerias capazes de fortalecer e auxiliar o Sistema Único de Saúde (SUS).

Para tanto, foram definidos dois importantes objetivos para o Satea: 1) proposição de atendimento ambulatorial interprofissional (Fonoaudiologia, Fisioterapia, Neurologia, Terapia Ocupacional, Psicologia e Neuropsicologia) para confirmação diagnóstica e construção do plano terapêutico singular; e 2) realização de atividade de educação permanente com profissionais de saúde e educação básica, especificamente com a equipe da Estratégia de Saúde da Família do município de Macaíba.

\section{TRABALHO INTERPROFISSIONAL E AÇÕES DE EDUCAÇÃO PERMANENTE EM SAÚDE: Processo de Instituição do Satea}

A população que ingressa no Satea é encaminhada pelas equipes de ESF, obedecendo os procedimentos utilizados pelo Sistema de Referência e Contrarreferência e pelo Sistema Nacional de Regulação (Sisreg). O Satea adotou um fluxo de atendimento interprofissional que proporciona a confirmação diagnóstica por meio de protocolos específicos de avaliação de características clínicas, cognitivas, comportamentais e de linguagem; assim como permite a construção do plano terapêutico singular do paciente com possibilidade de atendimento individualizado nas áreas de Fonoterapia, Fisioterapia, Terapia Sensorial (com integração sensorial), Psicoterapia e Equoterapia; ou ainda participação em grupos de orientação e suporte às famílias assistidas.

Também em prol de uma produção de saúde integral, o Satea adotou a Política Nacional de Humanização (PNH). Criada em 2005 pelo Ministério da Saúde, a PNH é uma política pública voltada para melhorar a qualidade dos serviços prestados pelo SUS. Designada como uma política pública transversal, a PNH propõe mudanças/melhorias dos modelos de atenção e gestão fundamentados na racionalidade biomédica, apresentando princípios que não dissociam a clínica da política e que adotam a transversalidade - ampliação das formas de conexão intra e intergrupos - como dispositivos para influenciar nos modos de atender os usuários e nos modos de gerir dos processos de trabalho (PASSOS, 2010)

Como diretrizes, convém destacar as orientações gerais para a inclusão de um método que engloba práticas como: a clínica ampliada, a cogestão dos serviços, a valorização do trabalho, o acolhimento, a defesa dos direitos do usuário, entre outras. Deste modo, a PNH contribui para potencializar inovadores processos de trabalho experienciados pela equipe interprofissional do Satea, valorizando a criação/ consolidação de estratégias construídas nos coletivos dedicados à promoção de mudanças nos modelos de atenção ao TEA (BARROS; SANTOS-FILHO, 2007).

Entre as estratégias, as ações de educação permanente em saúde com as equipes de ESF objetivam o desenvolvimento de novas competências e transformações nas práticas de atenção à saúde de crianças nos primeiros anos de vida com risco de TEA. Embora não haja, até o momento, evidência que respalde um diagnóstico no primeiro ano de vida da criança, é crescente o volume de conhecimentos sugerindo a possibilidade de identificação de risco de autismo nesse período. Assim, os Agentes Comunitários de Saúde e enfermeiros da Estratégia Saúde da Família (ESF) constituem-se como porta de entrada para rede do Sistema Único de Saúde e podem exercer papel essencial na observação de sinais precoces de autismo. Profissionais aptos à detecção precoce do autismo compactuam com a tendência atual de diagnóstico e tratamento precoces (antes dos 5 anos de idade).

\section{CONCEPÇÕES DE AGENTES \\ COMUNITÁRIOS DE SAÚDE E \\ ENFERMEIROS SOBRE O AUTISMO: O Primeiro Passo Para Construção Eficaz de Ações de Educação Permanente em Saúde Sobre o TEA}

O Ministério da Saúde adverte que a educação permanente requer elaboração, desenho e execução a partir de uma análise estratégica e da cultura institucional dos serviços de saúde em que se insere. Para se produzir mudança nas práticas e, sobretudo, para modificar práticas institucionalizadas nos serviços de saúde, é necessário privilegiar o conhecimento prático em suas ações educativas e favorecer a reflexão compartilhada e sistemática (ARAÚJO et al., 2017). 
Assim sendo, ações de educação permanente em saúde que garantam eficácia e efetividade exigem aproximar a educação da vida cotidiana e reconhecer o potencial educativo da situação de trabalho. A situação prevê transformar as situações diárias em aprendizagem, analisando reflexivamente os problemas da prática e valorizando o próprio processo de trabalho no seu contexto intrínseco. Esta perspectiva, centrada no processo de trabalho, implica perceber quais são os regimes discursivos e extradiscursivos vigentes, responsáveis por vincular determinados saberes, sobre os quais se exercem relações de poder e modos de operar na realidade posta (PASSOS, 2010)

Conforme estruturação da taxonomia de Bloom no domínio cognitivo (FERRAZ; BELHOT, 2010), a identificação do conhecimento prévio é o primeiro passo para facilitar a comunicação entre pessoas e atender às necessidades e diretrizes contextuais. Nessa perspectiva, o primeiro passo do Satea no processo de construção de ação de educação permanente em saúde foi a identificação das concepções de Agentes Comunitários de Saúde e enfermeiros sobre o autismo.

Participaram 54 ACS e 22 enfermeiros. Entre os agentes havia 37 homens e 17 mulheres, com idade média de 39 anos, o mais novo com 30 anos e o mais velho com 55. Entre os enfermeiros, 20 eram do sexo feminino e 2 do sexo masculino, com a idade média de 33 anos, o mais novo com 26 anos e o mais velho com 49. O tempo médio de atuação na ocupação profissional da atenção básica foi de 13 anos e meio para os Agentes Comunitários de Saúde e entre 5 anos e meio até 19 anos para os enfermeiros. Este cenário é representativo da realidade local do munícipio de Macaíba, no qual há 23 equipes da Estratégia Saúde da Família formadas, em sua maioria, por enfermeiros e Agentes Comunitários de Saúde.

A atividade de educação permanente realizada cumpriu o cronograma de 40 horas/aula com aulas mensais durante a semana e atendeu ao requisito institucional de realizar, no mínimo, $90 \%$ das ações de educação permanente no contexto da saúde materno-infantil programadas anualmente. $O$ instrumento utilizado para a obtenção dos dados foi um questionário estruturado em seis questões fechadas e uma aberta, abarcando o conhecimento dos profissionais nos seguintes domínios: causas do autismo, alterações comuns em autistas, características fundamentais, sinais de alerta para o autismo, idade para evidenciação dos sinais, categorias de atenção profissional ao autista e benefícios do rastreio precoce do autismo. As questões foram elaboradas considerando-se dados nosológicos e nosográficos constantes do CID-10, uma vez que este é o manual de referência utilizado pelo Sistema Único de Saúde. Esta etapa do trabalho foi aprovada pelo Comitê de Ética em Pesquisa da Universidade Federal do Rio Grande do Norte (CAAE: 11701312.9.0000.5537), cumprindo, portanto, com os aspectos éticos pertinentes à investigação envolvendo seres humanos, conforme a Resolução no 466/2012 do Conselho Nacional de Saúde (2012).

A aplicação do questionário ocorreu durante a realização de curso de educação permanente, denominado: "A importância da detecção precoce do autismo", realizado pelo Centro de Educação e Pesquisa em Saúde Anita Garibaldi, vinculado ao Instituto Santos Dumont, em parceria com a prefeitura de Macaíba. 0 questionário foi aplicado coletivamente, antes do início do curso. Os dados da pesquisa foram analisados por tratamento descritivo e qualitativo.

Os dados oriundos do questionário, entre as causas do autismo, $87,5 \%$ dos enfermeiros ao passo que $79,6 \%$ dos ACS apontaram ser a causa genética a principal razão para o autismo. $O$ dado referente a crises convulsivas também demonstrou um entendimento diferenciado entre as duas categorias profissionais: $3,3 \%$ dos ACS apontaram a crise convulsiva como causa para o autismo, ao passo que nenhum dos enfermeiros indicou essa causa para o autismo. Entre os enfermeiros, 12,5\% não associaram o autismo a nenhuma das causas anteriormente citadas.

No tocante às alterações comuns em autistas, $75 \%$ dos enfermeiros e $47,5 \%$ dos ACS optaram pela questão "todas as alternativas anteriores". Logo, ambas as categorias profissionais consideram os comprometimentos típicos do TEA, entre os quais alterações qualitativas na comunicação e interação social, movimentos repetitivos com o corpo e interesse restrito e intenso em determinado tema ou atividade. Dos ACS respondentes, 38,9\% destacaram o comprometimento na comunicação social como alteração exclusiva do TEA, mas apenas $8,3 \%$ dos enfermeiros tiveram a mesma concepção. Congênere, 32,2\% dos ACS destacaram os movimentos repetitivos com o corpo como alteração comum no autismo, mas apenas $12,5 \%$ dos enfermeiros apresentaram a mesma concepção.

Entre as características fundamentais da criança autista, $28,8 \%$ dos ACS e apenas $8,3 \%$ dos enfermeiros destacaram a presença de deficiência intelectual como característica fundamental da criança autista. Em relação à ausência de fala, $22 \%$ dos ACS assinalaram que a criança autista necessariamente não fala, enquanto que os enfermeiros não assinalaram essa característica como fundamental para identificação de uma criança autista. A falha no contato visual foi 
a característica de maior confluência entre ACS e enfermeiros, $75 \%$ e $44 \%$, respectivamente. Tais profissionais também confluem na concepção do comportamento agressivo: $16,6 \%$ dos enfermeiros e $15,2 \%$ dos ACS apontaram o comportamento agressivo como característica fundamental da criança autista.

Os resultados do domínio que investigou os sinais de alerta para o autismo foram os mais heterogêneos. Enfermeiros e ACS convergem sobre a importância da observação do brincar simbólico, mas divergem em relação à característica de não responder ao chamado dos pais/cuidador: $37,5 \%$ e $30,5 \%$, respectivamente, assinalaram o não brincar de faz-de-conta como sinal de alerta para o autismo; e 50\% e $30,5 \%$, respectivamente, consideraram o não responder ao chamado dos pais/cuidador como sinal de alerta para o autismo. Também foram heterogêneos os resultados acerca da idade em que se torna evidente os sintomas de autismo. Conforme observado, os dados apontam uma convergência para o primeiro ano de vida, mas dúvidas emergem quando se questiona a proeminência de sinais autistas antes ou depois de ingressar na escola.

Quanto aos cuidados essenciais à criança autista, $75 \%$ dos enfermeiros e $81,3 \%$ dos ACS apontaram a interação com familiares e colegas como ponto central no tratamento da criança autista. Apenas $8,3 \%$ dos enfermeiros e $5 \%$ dos ACS apostaram no medicamento como tratamento eficiente ao acompanhamento da criança autista.

\section{DAS CONCEPÇÕES SOBRE O AUTISMO À PRÁTICA COLABORATIVA ENTRE $O$ SATEA E AS EQUIPES DAS ESTRATÉGIAS DE SAÚDE DA FAMÍLIA}

Os Agentes Comunitários de Saúde constituem o elo entre a comunidade e o sistema de saúde. Eles são considerados atores nas trocas estabelecidas entre saberes populares de saúde e saberes médicos-científicos. Estudos clínicos atuais sobre o autismo apresentam evidências de correlação negativa entre a falta de rede de apoio familiar e emergência de sintomas autísticos (ESTES et al., 2013). A etiologia do autismo continua desconhecida, mas o grande número de respostas que vinculam o autismo à causa genética permite refletir sobre o papel que os enfermeiros e, em especial, os ACS exercem na comunidade para desmistificar possíveis saberes populares ainda vinculados à concepções sobre o autismo (MARANHÃO, 2014).
Ademais, a detecção do autismo é prioritariamente clínica. Profissionais e pesquisadores baseiam-se em um conjunto de características comportamentais, normalmente obtido pelo histórico clínico comportamental e observações clínicas. Frequentemente, pais ou cuidadores apresentam dificuldade para delinear tipos de características factíveis ao atraso ou funcionamento anormal da linguagem para fins de comunicação social antes dos 3 anos de idade. Comumente as interações sociais dos pequenos são restritas aos pais ou cuidadores, assim como padrões restritos e repetitivos de comportamentos, interesses e atividades não são tão óbvios no início da primeira infância. Tais padrões podem ser sucumbidos pela hiperatividade e, portanto, a equipe da ESF qualificada para detecção do TEA consegue alertar os pais para a procura de assistência clínica especializada.

Embora os ACS e enfermeiros identifiquem sinais comportamentais de alerta para o autismo, conforme apresentado, a escolha dos principais sinais comportamentais foi heterogênea e, algumas vezes, divergente entre enfermeiros e Agentes. Também foram heterogêneos os resultados acerca da idade em que se torna evidente os sintomas de autismo. Brentani et al. (2013) sugerem a árdua tarefa de identificação clínica do autismo ainda na primeira infância, notadamente quando a linguagem não apresenta atrasos importantes e o desempenho intelectivo é alto. Garcia e Lampreia (2011) apresentam os limites para a identificação de risco de autismo no primeiro ano, mas destacam as possibilidades para a identificação de risco a partir da análise de categorias de interação e de ordem afetiva que caracterizam as interações bebê-adulto nesse período. A identificação de comportamentos de risco de autismo, especialmente nos três primeiros anos de vida da criança, aumenta as chances de diagnóstico e intervenção precoces.

Os agentes comunitários e enfermeiros assinalaram que a criança autista necessariamente possui comportamentos agressivos, no entanto o comportamento agressivo não compõe critérios de identificação clínica do TEA. Estes et al. (2013) afirmam que associar o autismo à agressividade pode incorrer em dois problemas: dificuldade na identificação de possíveis casos de autismo - visto que o profissional espera encontrar a agressividade, mas tal comportamento não necessariamente estará presente - e posicionamentos deterministas equivocados quanto ao prognóstico dos pacientes diagnosticados. A agressão e heteroagressão podem se fazer presentes no autismo, mas não é regra. Conforme Mello (2013), faz-se necessária uma avaliação multiprofissional para compreender a(s) 
variável(is) motivacional(is) responsável(is) pela ocorrência do comportamento auto ou heteroagressivo. No autismo, quando presente, a agressão comumente está associada à ausência de estratégias eficazes para o estabelecimento da comunicação social ou ainda à necessidade de produzir efeitos sensoriais no corpo.

Concernente aos cuidados essenciais à criança autista, enfermeiros e agentes parecem cientes de que a medicação é uma alternativa possível para atenuar sintomas do autismo, mas constitui-se como tratamento complementar à intervenção multiprofissional especializada. A irritabilidade, autoagressividade, hiperatividade, insônia e comportamentos estereotipados podem ser melhorados com psicofármacos. Os médicos comumente procuram eleger os sintomas mais disfuncionais, ou seja, procuram um fármaco que atue seletivamente sobre aqueles sintomas que causam sofrimento à criança ou à família (SULLIVAN; STONE; DAWSON, 2014).

Conforme explicitado, os agentes e enfermeiros parecem compreender que os autistas e seus familiares também se beneficiam de intervenções clínicas não farmacológicas. A intervenção multiprofissional constitui-se como realidade necessária e fundamental para familiares e pacientes autistas. A precocidade do diagnóstico e a redução do estigma do diagnóstico são fundamentais, assim como a intervenção intensiva (não só em termos de carga horária, mas também de abrangência multiprofissional). Os estudos mais recentes sobre autismo destacam que intervenções precoces (antes dos 5 anos) são as mais efetivas e podem diminuir a necessidade de assistência especial para as faixas de idade seguintes (GARCIA; LAMPREIA, 2011).

Os dados obtidos no levantamento prévio sobre as concepções de Agentes Comunitários de Saúde e enfermeiros sobre o TEA foram essenciais para construção de uma jornada de práticas colaborativas entre o Satea e a comunidade. Foi possível verificar que os ACS e enfermeiros possuem informações sobre o autismo, mas estas precisam de aprimoramento, em especial acerca da identificação precoce dos sintomas.

Isso posto, associado ao contraponto entre maior prevalência de autismo nas últimas décadas e escassas políticas de atenção aos pacientes autistas e seus familiares, o Satea considerou pertinente instituir ações de educação permanente em saúde com o tema autismo. É preciso garantir formação adequada aos profissionais da atenção básica à saúde com o objetivo de auxiliar na identificação de sinais precoces de risco para o desenvolvimento infantil, assim como fornecer os direcionamentos adequados a cada caso. Para tanto, foi preciso pensar na educação per- manente como prática colaborativa de saúde entre a equipe interprofissional do Satea e a equipe das ESFs. Foi dada a largada ao início de uma jornada colaborativa, em que os profissionais mobilizam seus saberes e modos de agir a fim de permitir (re)construções de práticas sustentadas pelo cuidado permanente com as pessoas e sua comunidade.

\section{CONSIDERAÇÕES FINAIS}

A proposta foi documentar a experiência do trabalho interprofissional e de práticas colaborativos do Serviço de Atenção ao Espectro do Autismo (Satea), serviço vinculado ao Centro de Educação e Pesquisa em Saúde Anita Garibaldi, Instituto Santos Dumont, localizado no município de Macaíba (Rio Grande do Norte).

Atualmente o Satea constitui-se como de serviço de referência ambulatorial para a atenção à saúde da pessoa com TEA no município de Macaíba e demais cidades que compõem a 7ạ região de saúde do município de Natal, capital do Estado do Rio Grande do Norte, atendendo, aproximadamente, 200 crianças entre 0 e 12 anos com hipótese ou diagnóstico de TEA.

A atividade de educação permanente realizada cumpriu a missão do Instituto Santos Dumont (ISD), Organização Social da qual o Satea faz parte. O ISD é uma Organização Social (OS) financiada com recursos do Ministério da Educação (MEC). A OS atua na cidade de Macaíba (Rio Grande do Norte), nas áreas de educação, saúde materno-infantil, habilitação/reabilitação, neurociências e neuroengenharia. Sua missão é promover educação para a vida, formando cidadãos por meio de ações integradas de ensino, pesquisa e extensão com o fim de contribuir para a transformação mais justa e humana da realidade social brasileira.

O ISD apresenta duas unidades: O Instituto Internacional de Neurociências Edmond e Lily Safra (IIN-ELS), no qual há Programa de Pós-Graduação em Neuroengenharia, e o Centro de Educação e Pesquisa Saúde Anita Garibaldi (Ceps) no qual há educação e o trabalho interprofissional em saúde que buscam a reorientação da formação e do modelo de atenção à saúde na perspectiva da integralidade e da humanização do cuidado, da valorização dos usuários e suas necessidades como elementos fundamentais para o fortalecimento do Sistema Único de Saúde (SUS).

Nesta perspectiva, o Satea tentou cumprir o papel de reorientação da formação na perspectiva da integralidade e da humanização do cuidado, ao promover a educação permanente com os profissionais de saúde como ferramenta de diálogo e coconstrução 
do conhecimento em prol da detecção precoce do autismo a fim avançar no diagnóstico e intervenção precoces. Muito deste papel advém de um processo de trabalho pautado pela interprofissionalidade e pela construção de práticas colaborativas com as equipes locais de Estratégia de Saúde da Família. Reconhecemos que o trabalho interprofissional especializado e o diálogo permanente com a atenção básica fortalece a rede de cuidado com os familiares e pessoas com diagnóstico (ou hipótese) de TEA. Conforme discutido, os Agentes Comunitários de Saúde podem operar tanto na via da fiscalização dos riscos e controle da vida da população como na criação de redes microssociais de comunicação e troca, portanto fortalecer tais profissionais com conhecimento, pode fortalecer estratégias de cuidado à saúde de crianças com risco de desenvolvimento infantil ligado ao autismo.

O trabalho de educação permanente desenvolvido foi permeado por uma perspectiva de prática de saúde guiada pela corresponsabilidade e pelo estabelecimento de um vínculo que transformou o profissional de saúde em um ser implicado com o saber reconhecer o autismo. Para tanto, optou-se por aulas nas quais o acolhimento do saber do outro foi prioritário. Em uma dimensão dialógica, as aulas tinham espaço para uma escuta implicada pelo desejo de compreender a demanda sobre o autismo para, desse modo, poder produzir intervenções com finalidades adequadas à singularidade das representações sociocultural de como tal demanda é problematizada e posta pelo profissional em questão.

As aulas também foram pautadas pela integralidade, cuja plano de aula tentou: 1) integrar a demanda trazida pelo profissional ao contexto e ao ser biopsicossocial com hipótese de autismo; 2 ) integrar a demanda a outros profissionais da saúde a fim de promover a interseção de saberes para intervenções eficazes; 3) integrar a demanda ao sistema público de saúde a fim de permitir que o profissional tivesse acesso a todas as possibilidades de práticas de saúde e contato com a diversidade de tecnologias que a rede de saúde pode oferecer para a resolução da detecção precoce do autismo. Desse modo, com esta concepção de cuidado, a atividade de educação permanente realizada tentou elucidar a necessidade de sermos profissionais pensantes e preocupados com a (re) construção permanente de práticas de saúde.

Esta experiência também tem favorecido o acesso dos profissionais locais à educação permanente, reconhecendo-os como agentes fundamentais para o aprendizado em serviço e para a legitimação do papel formador do SUS. A experiência em curso refe- renda a potência da interprofissionalidade ao cuidado do TEA, no entanto o impacto na comunidade clínica só poderá ser avaliado, efetivamente, em longo prazo, com outras atividades de educação permanente e mensuração dos conhecimentos consolidados.

Para finalizar, compreende-se que as experiências aqui apresentadas podem servir de base para outros serviços especializados na saúde materno-infantil e na reabilitação, além, é claro, de oferecer aos usuários do SUS e integrantes do Instituto Santos Dumont a documentação do processo de criação e instituição de um serviço especializado no atendimento do TEA na região do Nordeste do Brasil, o que, em última instância, permite avaliar o percurso, redefinir planos e redirecionar os caminhos.

\section{REFERÊNCIAS}

ABREU, Camila Soares de; CARDOSO-MARTINS, Cláudia; BARBOSA, Poliana Gonçalves. A Relação entre a atenção compartilhada e a teoria da mente: um estudo longitudinal. Psicologia: Reflexão e Crítica, v. 27, n. 2, p. 409-414, 2014. Fap Unifesp (SciELO). http://dx.doi.org/10.1590/16787153.201427222.

ALEIXO, J. L. M. A Atenção Primária à Saúde e o Programa de Saúde da Família: perspectivas de desenvolvimento no início do terceiro milênio. Revista Mineira de Saúde Pública, Minas Gerais, v. 1, n. 1, p. 1-16, jan. 2013.

AMERICAN PSYCHIATRIC ASSOCIATION (org.). DSM-5: manual diagnóstico e estatístico de transtornos mentais. 5. ed. Porto Alegre: Artmed, 2014.

ARAÚJO, T. A. M. de et al. Multiprofissionalidade e interprofissionalidade em uma residência hospitalar: o olhar de residentes e preceptores. Interface - Comunicação, Saúde, Educação, v. 21, n. 62, p. 601-613, 23 jan. 2017. Fap Unifesp (SCiELO). http://dx.doi.org/10.1590/1807-57622016.0295.

BARROS, M. E. B. de; SANTOS-FILHO, S. B. (org.). Trabalhador da saúde: muito prazer! Protagonismo dos trabalhadores na gestão do trabalho em saúde. ljuí, RS: Editora Unijuí, 2007. 272p.

BOSA, Cleonice. Atenção compartilhada e identificação precoce do autismo. Psicologia: Reflexão e Crítica, Porto Alegre, 2002. p. 77-88. Semestral.

BRASIL. Constituição (1988). Lei no 196, de 7 de abril de 1988. A saúde é direito de todos e dever do Estado, garantido mediante políticas sociais e econômicas que visem à redução do risco de doença e de outros agravos e ao acesso universal e igualitário às ações e serviços para sua promoção, proteção e recuperação. VII da Ordem Social. Brasília, DF, Seção 2.

BRENTANI, H. et al. Autism spectrum disorders: an overview on diagnosis and treatment. Revista Brasileira de Psiquiatria, v. 35, n. 1, p. 62-72, 2013. Fap Unifesp (SciELO). http:// dx.doi.org/10.1590/1516-4446-2013-s104. 
CAMARGO-BORGES, C.; CARDOSO, C. L. A psicologia e a estratégia saúde da família: compondo saberes e fazeres. Psicologia \& Sociedade, v. 17, n. 2, p. 26-32, ago. 2005. Fap Unifesp (SciELO). http://dx.doi.org/10.1590/s010271822005000200005.

CHINELLO, Alice; GANGI, Valentina di; VALENZA, Eloisa. Persistent primary reflexes affect motor acts: Potential implications for autism spectrum disorder. Research In Developmental Disabilities, v. 83, p. 287-295, Dec. 2018. Elsevier BV. http://dx.doi.org/10.1016/j.ridd.2016.07.010.

DAVID, Maude M.; BABINEAU, Brooke A.; WALL, Dennis P. Can we accelerate autism discoveries through crowdsourcing? Research In Autism Spectrum Disorders, v. 32, p. 80-83, Dec. 2016. Elsevier BV. http://dx.doi.org/10.1016/j. rasd.2016.09.001.

ESTES, A. et al. The Impact of Parent-Delivered Intervention on Parents of Very Young Children with Autism. Journal of Autism and Developmental Disorders, v. 44, n. 2, p. 353-365, 10 July 2013. Springer Nature. http://dx.doi.org/10.1007/ s10803-013-1874-z.

FERRAZ, A. P. do C. M.; BELHOT, R. V. Taxonomia de Bloom: revisão teórica e apresentação das adequações do instrumento para definição de objetivos instrucionais. Gestão \& Produção, v. 17, n. 2, p. 421-431, 2010. Fap Unifesp (SciELO). http://dx.doi.org/10.1590/s0104-530x2010000200015.

GARCIA, M. L.; LAMPREIA, C. Limites e possibilidades da identificação de risco de autismo no primeiro ano de vida. Psicologia: Reflexão e Crítica, Porto Alegre, v. 24, n. 2, p. 300-308, dez. 2011. Fap Unifesp (SciELO). http://dx.doi. org/10.1590/S0102-79722011000200011.

GILLET, Patrice. Neuropsicologia do autismo na criança. Lisboa: Piaget, 2014.

HOPKINS, D. (ed.). Marco para ação em educação interprofissional e prática colaborativa. Genebra: Freelance, 2010.

LANCETTI, A. A potência terapêutica dos agentes comunitários de saúde. In: LANCETTI, A. Clínica peripatética. 3. ed. São Paulo: Hucitec, 2008. p. 1-127. Cap. 5.

MARANHÃO, S. Caracterização de aspectos da cognição social, habilidades sociais e funções executivas de crianças diagnosticadas com Transtorno Autista e Transtorno de Asperger. 2014, 264f. Dissertação (Mestrado) - Universidade Federal do Rio Grande do Norte, Curso de Psicologia, Programa de Pós-Graduação em Psicologia, Natal, 2014.
MELLO, A. M. S. R. de. Retratos do autismo no Brasil. São Paulo: Ama, 2013.

NOGUEIRA, Santos; SEABRA, Karla; MOURA, Maria de Lourdes Seidl de. Diagnóstico precoce de autismo e desenvolvimento infantil inicial: um estudo comparativo de dois bebês com um mês de vida. In: REUNIÃO ANUAL DE PSICOLOGIA, 30., 2000, Brasília. Anais [...]. Brasília: SBP, 2000. p. 1-138.

PASSOS, E. (org.). Cadernos Humaniza SUS. Brasília: Ministério da Saúde, 2010. 1 v.

PIERCE, Karen et al. Preference for Geometric Patterns Early in Life as a Risk Factor for Autism. Archives of General Psychiatry, v. 68, n. 1, p. 101-109, 3 Jan. 2011. American Medical Association (AMA). http://dx.doi.org/10.1001/archgenpsychiatry.2010.113.

SCHMIDT, Carlo. Transtorno do Espectro Autista: onde estamos e para onde vamos. Psicologia em Estudo, Universidade Estadual de Maringá,. v. 22, n. 2, p. 221-230, 2 jul. 2017. http://dx.doi.org/10.4025/psicolestud.v22i2.34651.

SRINIVASAN, Sudha M.; BHAT, Anjana N. Differences in object sharing between infants at risk for autism and typically developing infants from 9 to 15 months of age. Infant Behavior And Development, v. 42, p. 128-141, Feb. 2016. Elsevier BV. http://dx.doi.org/10.1016/j.infbeh.2015.12.003.

SULLIVAN, K.; STONE, W. L.; DAWSON, G. Potential neural mechanisms underlying the effectiveness of early intervention for children with autism spectrum disorder. Research in Developmental Disabilities, v. 35, n. 11, p. 2.9212.932, Nov. 2014. Elsevier BV. http://dx.doi.org/10.1016/j. ridd.2014.07.027.

SURJUS, L. T. de L. e S. (org.). Linha de cuidado para a atenção às pessoas com transtornos do espectro do autismo e suas famílias na Rede de Atenção Psicossocial do Sistema Único de Saúde. Brasília: Ministério da Saúde, 2015. 157p. Disponível em: http://editora.saude.gov.br/. Acesso em: 9 abr. 2018.

ZAQUEU, Lívia da Conceição Costa et al. Associações entre sinais precoces de autismo, atenção compartilhada e atrasos no desenvolvimento infantil. Psicologia: Teoria e Pesquisa, v. 31, n. 3, p. 293-302, set. 2015. Fap Unifesp (SciELO). http://dx.doi.org/10.1590/0102-37722015032243293302.

ZILBOVICIUS, Monica et al. Autism, the superior temporal sulcus and social perception. Trends In Neurosciences, v. 29, n. 7, p. 359-366, July 2006. Elsevier BV. http://dx.doi.org/10.1016/j.tins.2006.06.004. 